\title{
Correction to: Shared genetic architecture between metabolic traits and Alzheimer's disease: a large-scale genome-wide cross-trait analysis
}

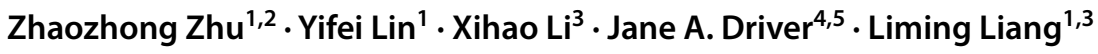

Published online: 10 November 2020

○) Springer-Verlag GmbH Germany, part of Springer Nature 2020

\section{Correction to: Human Genetics (2019) 138:271-285 https://doi.org/10.1007/s00439-019-01988-9}

Page 4: In the "Results-Genetic correlation between AD and metabolic traits" section, the sentence "We did not observe substantial genetic correlation between $\mathrm{AD}$ and obesity traits (BMI and WHR, both $\mathrm{Rg}<0.05$ and $P>0.3$ ), T2D $(\mathrm{Rg}=0.106, P>0.2)$ and other lipid traits $(\mathrm{LDL}, \mathrm{TC}$ and TG, $\mathrm{Rg}=0.104,-0.076,0.022$, respectively, all $P>0.17$ ) (Table 1)" should be "We did not observe substantial genetic correlation between $\mathrm{AD}$ and obesity traits (BMI and WHR, both $\operatorname{Rg}<0.05$ and $P>0.3)$, T2D $(\operatorname{Rg}=0.106, P>0.2)$ and other lipid traits (LDL, TC and TG, $\mathrm{Rg}=-0.178,-0.108$, - 0.051, respectively, all $P>0.39$ ) (Table 1)".

Page 4: In the "Results-Genetic correlation between AD and metabolic traits" section, the sentence "We also observed that HDL had a significant genetic correlation with AD $(\mathrm{Rg}=-0.137, P=0.0436)$ " should be "We also observed

The original article can be found online at https://doi.org/10.1007/ s00439-019-01988-9.

Liming Liang

lliang@hsph.harvard.edu

1 Program in Genetic Epidemiology and Statistical Genetics, Department of Epidemiology, Harvard T.H. Chan School of Public Health, Boston, MA, USA

2 Department of Environmental Health, Harvard T.H. Chan School of Public Health, Boston, MA, USA

3 Department of Biostatistics, Harvard T.H. Chan School of Public Health, Boston, MA, USA

4 Geriatric Research Education and Clinical Center and Massachusetts Veterans Epidemiology Research and Information Center, VA Medical Center, Boston, MA, USA

5 Division of Aging, Brigham and Women's Hospital, Harvard Medical School, Boston, MA, USA that HDL had a significant genetic correlation with AD $(\mathrm{Rg}=0.322, P=0.017)$ ".

Page 4: In the "Results-Analysis of partitioned genetic correlation by functional category section", the sentence "In addition, the transcribed region was found to have the highest level of genetic correlation between AD and HDL $(\mathrm{Rg}=-0.1471)$ " should be "In addition, the transcribed region was found to have the highest level of genetic correlation between $\mathrm{AD}$ and HDL $(\mathrm{Rg}=0.261)$ ".

Page 9: In the second paragraph of the Discussion section, the sentence "Our findings suggest that the phenotypic correlation between $\mathrm{AD}$ and metabolic traits was due to a common genetic predisposition base" should be "Our findings suggest that the phenotypic correlation between AD and metabolic traits was due to a common genetic predisposition base, however, may not be in the same direction".

Page 9: In the second paragraph of the Discussion section "In the analysis of partitioned co-heritability by functional categories, we observed positive genetic correlation between $\mathrm{AD}$ and fasting glucose, FG-FINS meta-analysis" should be "In the analysis of partitioned co-heritability by functional categories, we observed positive genetic correlation between $\mathrm{AD}$ and fasting glucose, FG-FINS meta-analysis or HDL".

Page 9: In the Discussion second paragraph, the sentence "and genetic correlation estimates were significant in transcribed, TFBS, super enhancer, H3K4me1, H3K27ac and DGF in AD-HDL trait pair "(Fig. 1b)" should be "and genetic correlation estimates were significant in transcribed, TFBS, super enhancer, H3K4me3, H3K4me1, FetalDHS, DHS and DGF in AD-HDL trait pair (Fig. 1b)". 